OPEN ACCESS

Edited by:

Tatjana Rundek

University of Miami, United States

Reviewed by:

Ilaria Di Donato,

University of Siena, Italy

Yuishin Izumi,

Tokushima University, Japan

*Correspondence:

Seung-Hun Oh

ohsh72@chamc.co.kr

†These authors have contributed equally to this work and share first

authorship

Specialty section:

This article was submitted to

Stroke,

a section of the journal

Frontiers in Neurology

Received: 11 October 2019

Accepted: 07 December 2020

Published: 18 January 2021

Citation:

Woo M-H, Lee KO, Chung D,

Choi J-W, Kim S-H and Oh S-H

(2021) Triglyceride/HDL-Cholesterol

Ratio as an Index of Intracranial

Atherosclerosis in Nonstroke Individuals. Front. Neurol. 11:504219.

doi: 10.3389/fneur.2020.504219

\section{Triglyceride/HDL-Cholesterol Ratio as an Index of Intracranial Atherosclerosis in Nonstroke Individuals}

\author{
Min-Hee Woo ${ }^{1 \dagger}$, Kee Ook Lee ${ }^{1+}$, Darda Chung ${ }^{1}$, Jung-Won Choi ${ }^{1}$, Sang-Heum Kim ${ }^{2}$ and \\ Seung-Hun $\mathrm{Oh}^{1 *}$
}

${ }^{1}$ Department of Neurology, CHA Bundang Medical Center, CHA University, Seongnam, South Korea, ${ }^{2}$ Department of Radiology, CHA Bundang Medical Center, CHA University, Seongnam, South Korea

Background: Triglyceride (TG)/high-density lipoprotein cholesterol ratio (THR) is a marker of dyslipidemia, and high THR is associated with an increase in cardiovascular events. In the present study, whether THR was associated with various markers of cerebral vascular pathologies, atherosclerosis of major cerebral arteries, including large artery atherosclerosis (LAA) and cerebral small vessel disease (SVD), in neurologically healthy individuals was investigated.

Methods: Vascular risk factors, brain magnetic resonance imaging (MRI) scans, and MR angiograms of 851 study subjects were evaluated. Findings of extracranial atherosclerosis (ECAS) and intracranial atherosclerosis (ICAS) were considered indices of LAA based on brain MR angiograms. The presence of silent lacunar infarct (SLI) and white matter hyperintensities (WMHs) were evaluated as indices of SVD based on brain MRIs.

Results: Subjects with ICAS (odds ratio, 1.83; 95\% confidence interval, 1.06-3.16; $P$ $=0.03)$ were significantly more likely to have high THR tertile $(T H R>2.06)$ than low THR tertile $(T H R<1.37)$ after adjusting for cardiovascular risk factors. THR was higher in subjects with multiple ICAS lesions than in those with single ECAS or without ICAS lesions. Associations among THR tertiles in ECAS, SLI, and WMHs were not significant.

Conclusion: In the present study, a positive association between high THR and the development of ICAS was observed in neurologically healthy participants.

Keywords: triglyceride, high-density lipoprotein, intracranial atherosclerosis, large artery atherosclerosis, small vessel disease, hyperlipidemia

\section{INTRODUCTION}

Modification of vascular risk factors is important for future stroke prevention. Ischemic stroke is caused by heterogeneous vascular pathologies in the brain (1). Atherosclerosis of major cerebral arteries is one of the strongest risk factors for ischemic stroke. The large artery atherosclerosis (LAA) is divided into extracranial atherosclerosis (ECAS) and intracranial atherosclerosis (ICAS) based on the anatomical site of atherosclerosis. In recent studies, ECAS and ICAS were reported to differ based on ethnic background and pathogenesis (2-4). 
Cerebral small vessel diseases (SVDs), such as silent lacunar infarct (SLI) and cerebral white matter hyperintensities (WMHs), are other risk factors for ischemic stroke (5). SVD is caused by occlusion of small perforating arteries or arterioles in the brain (6). Although SVDs share common risk factors with LAA, the pathogenesis is distinct from LAA (7). These findings indicate novel factors besides classical vascular risk factors for ischemic stroke may be involved in the distinct break cerebrovascular pathology.

Hyperlipidemia, usually defined as high level of serum lowdensity lipoprotein cholesterol (LDL-C), is a risk factor for coronary arterial atherosclerotic disease. Statins can significantly decrease (up to 37\%) the incidence of cardiovascular disease (8). Serum LDL-C levels are also a risk factor for ischemic stroke. The Stroke Prevention by Aggressive Reduction in Cholesterol Levels trial in patients with recent stroke or transient ischemic attack clearly showed that intensive lowering of LDL-C using statin treatment reduced the overall incidence of strokes and cardiovascular events (9). However, residual cardiovascular risk remains high despite significant effects of LDL-C lowering therapy for prevention of ischemic stroke. Because many patients with ischemic stroke still have a high residual risk of recurrent stroke or cardiovascular events despite extensive LDL-C lowering treatment, additional lipid modifications beyond controlling traditional vascular risk factors are needed to reduce the residual risk.

Triglyceride (TG) and high-density lipoprotein (HDL-C) are lipid elements that are commonly tested in clinical setting. Several studies have shown that high level of TG contributes to the development and progression of atherosclerosis and risk for ischemic stroke (10-12). Low level of HDL-C increases the rate of ischemic stroke (13) and carotid stenosis (14).

Recently, the LDL particle phenotype has attracted more attention than the total amount of LDL-C because small, dense LDL particles are highly atherogenic. Atherogenic dyslipidemia, defined as high level of serum TG, high level of small/dense LDL particles, and low level of HDL-C, evidently increases cardiovascular disease (15). Furthermore, the simultaneous use of TG and HDL-C has been found to be more useful than isolated lipid levels because this level closely reflects the complex interactions of lipoprotein metabolism (16). The TG and HDLC level ratio (THR) was shown directly correlated with small, dense LDL particles in several studies, indicating THR could be feasibly used as an index of atherogenic dyslipidemia in the clinical setting (17-19). In addition, high THR is associated with insulin resistance, metabolic diseases, and cardiocerebrovascular diseases (20-22).

However, whether THR is associated with different cerebrovascular pathologies remains unknown. In several studies, a positive association was observed between THR and LAA $(23,24)$; however, other research has shown an association between THR and SVDs (25). Moreover, the association of THR with four distinct cerebrovascular pathologies (ECAS, ICAS, SLI, and WMH) in a single cohort has not been investigated. In the present study, the association of THR with different cerebrovascular pathologies in nonstroke individuals was investigated.

\section{PATIENTS AND METHODS}

\section{Study Population and Protocol}

The study was a retrospective analysis of neurologically healthy individuals who visited the outpatient clinic of the Department of Neurology or Healthcare Center in the CHA Bundang Medical Center between March 2008 and December 2014. All subjects presented for routine health examinations or medical attention due to underlying cardiovascular risk factors. Briefly, only individuals ranging from 50 to 79 years of age who underwent brain magnetic resonance imaging (MRI) and magnetic resonance angiography (MRA) were enrolled. The medical records, laboratory tests results, and radiological findings were reviewed. The study included only subjects whose records contained adequate demographic, radiological, and laboratory data. Among 959 study subjects, 108 were excluded for the following reasons: inadequate medical information $(n=22)$, no laboratory tests performed $(n=74)$, and previous history of neurological disease $(n=12)$. A total of 851 subjects were included in this study. Each subject's data were deidentified prior to analysis. The institutional review board (IRB) of CHA Bundang Medical Center approved the study (IRB no. BD-2010083).

\section{Diagnostic Criteria for Clinical Characteristics}

Hypertension was diagnosed if the subject had a systolic blood pressure $\geq 140 \mathrm{~mm} \mathrm{Hg}$ or a diastolic blood pressure $\geq 90 \mathrm{~mm}$ $\mathrm{Hg}$ on repeated measurements, or the subject was taking antihypertensive medication. Diabetes mellitus was diagnosed if the subject had a fasting plasma glucose $\geq 7.0 \mathrm{mmol} / \mathrm{L}$ or was taking antidiabetic medications or insulin. Hypercholesterolemia was diagnosed if the subject had a total cholesterol $\geq 5.69$ $\mathrm{mmol} / \mathrm{L}$ or was taking lipid-lowering agents. Current smoking was defined if the subject had smoked within 1 year prior to examination. Coronary arterial occlusive disease (CAOD) was diagnosed if subjects had a history of acute myocardial infarction, unstable angina, CAOD confirmed by angiography, or coronary surgery or intervention. Chronic kidney disease $(\mathrm{CKD})$ was defined as an estimated glomerular filtration rate $<60$ $\mathrm{mL} / \mathrm{min} / 1.73 \mathrm{~m}^{2}$.

\section{Measurement of Blood Parameters and THR}

Laboratory data collected for analysis included serum fasting glucose, total cholesterol, HDL-C, and TG. The THR was calculated as TG divided by HDL-C. To evaluate the factors associated with THR, subjects were divided into three groups based on serum THR tertile: (1) low THR tertile group (T1), THR $<$ 1.37; (2) middle THR tertile group (T2), THR ranging from 1.37 to 2.06; and (3) high THR tertile group (T3), THR $>2.06$.

\section{Diagnostic Criteria for Cerebral Atherosclerosis and Cerebral SVDs}

Brain MRI and MRA were performed using one of three 1.5-T MR systems (Sonata, Siemens Healthcare, $n=568$; Signa Excite, GE Healthcare, $n=88$; Signa HDx, GE Healthcare, $n=95$ ). 
A radiologist who was blinded to clinical and laboratory data assessed the images. ECAS and ICAS were evaluated as MR indices of LAA based on the location of atherosclerotic lesions visualized using MRA. The ECAS was defined as stenosis $\geq 50 \%$ in the external cranial portion of the internal carotid artery or vertebral artery on gadolinium contrast-enhanced MRA, using methods described in the North American Symptomatic Carotid Endarterectomy Trial study (26). The ICAS was defined as stenosis $\geq 50 \%$ in the proximal portions of the middle cerebral artery, anterior cerebral artery, posterior cerebral artery, intracranial portion of the vertebral artery, and basilar artery based on time-of-flight image, using the method described in the Warfarin vs. Aspirin for Symptomatic Intracranial Disease study (27). Normal arterial variations, such as a unilateral origination of the bilateral anterior cerebral arteries or fetal-type posterior cerebral artery, were not regarded as true atherosclerosis.

Next, SLI and WMHs were evaluated as MR indices of SVD visualized on brain MRI. The SLI was defined as a small (3$15 \mathrm{~mm}$ in diameter) cavity-like lesion in an area of low signal intensity on T1-weighted images [repetition time (TR)/echo time $(\mathrm{TE})=560 / 14 \mathrm{~ms}]$. The WMH was defined as a hyperintense lesion in an area of bilateral cerebral white matter visualized on a fluid-attenuated inversion recovery image $(\mathrm{TR} / \mathrm{TE}=$ 9,000/105 ms, inversion time, 2,500 ms). The degree of the WMH was scored using the visual grading method proposed by Fazekas et al. (28). Periventricular and deep subcortical white matter scores were added (ranging from 0 to 6 points), and lesions with a score $\geq 3$ points were regarded as WMHs in the brain.

\section{Statistical Analyses}

To evaluate the factors associated with THR tertile group, a between-group comparison was conducted using analysis of variance (ANOVA) for continuous variables and $\chi^{2}$ test for categorical variables. To evaluate the independent association of ECAS, ICAS, SLI, or WMH across THR tertile groups, binary logistic regression analyses were performed between each group with the low THR tertile group as a reference group. Potential confounding factors were adjusted including age, gender, hypertension, diabetes, statin medication, current smoking, CAOD, CKD, and other significant variables obtained from univariate analysis. Odds ratio (OR) and 95\% confidence interval (CI) were calculated. All statistical analyses were performed using the $\mathrm{R}$ package for Windows (version 3.1.3; $\mathrm{R}$ Foundation for Statistical Computing, Vienna, Austria). A two-sided $P<0.05$ was considered statistically significant.

\section{RESULTS}

The mean age of the 851 study subjects was $65.2 \pm 8.8$ years, and $60.4 \%$ were females. The mean THR was $1.5 \pm 1.5$. The prevalence of ECAS, ICAS, SLI, and WMHs was 14.2, $13.6,16.6$, and $30.6 \%$, respectively. The distributions of several baseline characteristics of the entire study population across THR tertiles are shown in Table 1. The prevalence of ICAS was significantly different among THR tertile groups, with a higher THR associated with a more frequent prevalence of ICAS $(P=$ 0.048 , Table 1). The prevalence of ECAS, SLI, and WMHs did not differ among THR tertile groups. Logistic regression analyses were performed to compare ECAS, ICAS, SLI, and WMHs across THR tertile groups after adjusting for confounding factors (age, gender, hypertension, diabetes mellitus, smoking status, statin medication, CAOD, and CKD; Table 2). The prevalence of ICAS was significantly higher in the high THR group (T3) than in the low THR tertile group (T1) (OR, 1.83; 95\% CI, 1.06-3.16; P $=0.03)$. The prevalence of ICAS was not significantly different between the middle and low THR tertile groups. The prevalence of ECAS, SLI, and WMHs did not differ among THR tertile groups based on multivariate logistic regression analysis.

In the logistic regression analysis to evaluate the individual risk factors associated with LAA and SVD indices, a significant association was observed between THR and ICAS (OR, 1.24; 95\% CI, 1.10-1.39; $P=0.001)$. Other significant variables for ICAS were older age and high prevalence of hypertension. THR showed no significant association in subjects with ECAS, SLI, and WMHs. Significant variable for ECAS was high prevalence of diabetes mellitus. Significant variables for SLI were older age, a high prevalence of hypertension, and current smoker. Significant variables for WMH were older age, a high prevalence of hypertension, and CKD (Table 3).

The effects of THR on the number of ICAS lesions were investigated in patients without ICAS lesion $(n=734)$, patients with single ICAS lesion $(n=59)$, and patients with multiple ICAS lesions $(n=57)$. Statistical significant difference was observed in the THR among the three groups based on ANOVA $(F=14.03, P$ $<0.001)$. The post hoc analysis showed that subjects with multiple ICAS lesions had higher THR $(2.55 \pm 3.09)$ than subjects without ICAS lesions $(1.47 \pm 1.29)$ and subjects with a single ICAS lesion $(1.61 \pm 1.37$; Figure 1).

\section{DISCUSSION}

In the present study, THR was closely associated with ICAS. After adjusting for confounding factors, subjects with high THR tertile displayed $83 \%$ higher prevalence of ICAS compared with subjects with low THR tertile. Conversely, THR was not associated with other cerebrovascular pathologies including ECAS and SVD. In agreement with our findings, Sirimarco et al. reported that symptomatic ICAS was strongly associated with low HDL-C and high TG levels (24). In addition, Kang et al. demonstrated that THR was associated with the presence of a steno-occlusive lesion in the basilar artery (23). These results indicate THR as an index of atherogenic dyslipidemia is a useful marker for ICAS, and different pathogenesis exists between ICAS and other cerebrovascular pathologies.

Although the mechanism of the differential impact of THR on ICAS and ECAS is unclear, several explanations are possible. In recent studies, EC and IC arteries were shown to have different metabolic and biological properties. The IC arteries have higher antioxidant capacity and become more prone to oxidative stress than EC arteries (29). HDL-C has antioxidant activity, which inhibits the oxidation of phospholipids and the activity of LDL-C (30). Low HDL-C possibly increases the oxidative stress in the more vulnerable IC arteries than 
TABLE 1 | Clinical characteristics of subjects based on THR tertiles.

\begin{tabular}{|c|c|c|c|c|c|}
\hline & $\begin{array}{c}\text { ALL } \\
(n=851)\end{array}$ & $\begin{array}{c}\text { T1 } \\
(n=283)\end{array}$ & $\begin{array}{c}\text { T2 } \\
(n=284)\end{array}$ & $\begin{array}{c}\text { T3 } \\
(n=284)\end{array}$ & $P$ value \\
\hline Gender (female, \%) & $514(60.4)$ & $190(67.1)$ & $180(63.4)$ & $144(50.7)$ & $<0.001$ \\
\hline Age, years & $65.2 \pm 8.8$ & $65.7 \pm 9.1$ & $65.7 \pm 9.1$ & $64.2 \pm 8.1$ & 0.072 \\
\hline Hypertension (\%) & $470(55.2)$ & $136(48.1)$ & $159(56.0)$ & $175(61.6)$ & 0.005 \\
\hline Diabetes mellitus (\%) & 209 (24.6) & $58(20.5)$ & $66(23.2)$ & 85 (29.9) & 0.027 \\
\hline CAOD (\%) & $62(7.3)$ & $17(6.0)$ & $22(7.7)$ & $23(8.1)$ & 0.591 \\
\hline CKD (\%) & $159(18.8)$ & $45(16.0)$ & $49(17.4)$ & $65(23.0)$ & 0.077 \\
\hline Statin medication (\%) & $174(20.4)$ & 47 (16.6) & $51(18.0)$ & $76(26.8)$ & 0.005 \\
\hline SBP, mm Hg & $131.0 \pm 18.9$ & $130.7 \pm 20.6$ & $130.9 \pm 20.0$ & $131.6 \pm 15.6$ & 0.842 \\
\hline DBP, mm Hg & $80.0 \pm 11.5$ & $79.6 \pm 12.4$ & $79.8 \pm 11.6$ & $80.6 \pm 10.6$ & 0.562 \\
\hline HDL-C, mmol/L & $1.2 \pm 0.3$ & $1.5 \pm 0.3$ & $1.2 \pm 0.2$ & $1.0 \pm 0.2$ & $<0.001$ \\
\hline $\mathrm{TG}, \mathrm{mmol} / \mathrm{L}$ & $1.7 \pm 1.2$ & $0.8 \pm 0.2$ & $1.4 \pm 0.3$ & $2.8 \pm 1.5$ & $<0.001$ \\
\hline \multicolumn{6}{|l|}{ LAA } \\
\hline ECAS (\%) & $121(14.2)$ & $36(12.7)$ & $40(14.1)$ & 45 (15.9) & 0.554 \\
\hline ICAS (\%) & $116(13.6)$ & $28(9.9)$ & $40(14.1)$ & $48(17.0)$ & 0.048 \\
\hline \multicolumn{6}{|l|}{ SVD } \\
\hline SLI (\%) & $141(16.6)$ & $48(17.0)$ & $43(15.1)$ & $50(17.6)$ & 0.715 \\
\hline WMH (\%) & $260(30.6)$ & $90(31.8)$ & $791(27.8)$ & $91(32.0)$ & 0.471 \\
\hline
\end{tabular}

Values are presented as percentages or means \pm standard deviation. $P$ values were derived from an analysis of variance (ANOVA) across THR tertile groups. T1, T2, and T3 represent low, middle, and high THR tertile groups, respectively. THR, TG/HDL-C ratio; CAOD, coronary arterial occlusive disease; CKD, chronic kidney disease; SBP, systolic blood pressure; DBP, diastolic blood pressure; eGFR, estimated glomerular filtration rate; HDL-C, high-density lipoprotein cholesterol; TG, triglyceride; LAA, atherosclerosis of major cerebral arteries; SVD, small vessel disease; ECAS, extracranial atherosclerosis; ICAS, intracranial atherosclerosis; SLI, silent lacunar infarct; WMH, white matter hyperintensity; OR, odds ratio; Cl, confidence interval.

TABLE 2 | Logistic regression analysis of ECAS, ICAS, SLI, and WMH based on THR tertiles.

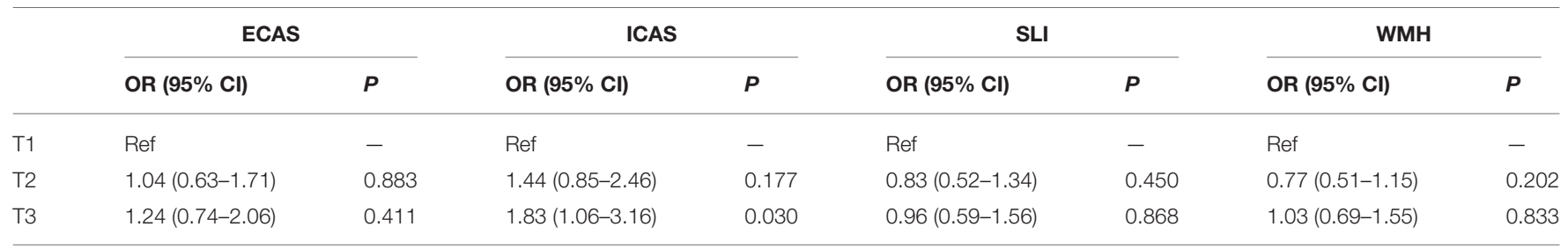

The analyses were conducted after adjusting age, gender, hypertension, diabetes mellitus, smoking status, statin medication, CAOD, and CKD.

T1, T2, and T3 represent low, middle, and high THR tertile groups, respectively.

Ref represents the low THR tertile group (T1) as a reference group for analyses.

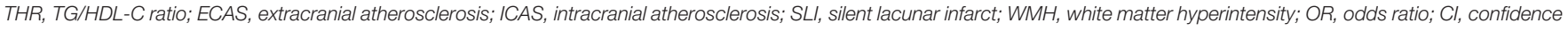
interval; $C A O D$, coronary arterial occlusive disease; $C K D$, chronic kidney disease.

EC arteries, which leads to development of ICAS. Another explanation is the closed link between metabolic syndrome (MetS) and ICAS. In several studies, MetS was more specifically associated with ICAS than ECAS (31-33). Because both TG and HDL-C are indicative of MetS, high THR may contribute to development of ICAS. In addition, MetS is associated with insulin resistance and increases the patient's vulnerability to oxidative stress (34). Insulin resistance was also associated with ICAS in nondiabetic patients (35). TG and HDL-C are independently associated with insulin resistance (36) but are more likely to be caused by abnormalities unrelated to insulin resistance (37). Therefore, THR may be a physiologically more relevant choice than HDL-C or TG alone. In addition, elevated TG and reduced HDL-C levels facilitate the formation of atherogenic LDL particles (19). Because of pathophysiological characteristics of both adventitia and media of the intracranial arteries, small, dense LDL particles penetrate the arterial wall more easily and bind to proteoglycans, rendering the arteries more susceptible to oxidative modifications $(24,38)$. Overall, the combination of these two conditions (elevated TG and reduced HDL-C levels) 
TABLE 3 | Logistic regression analysis of vascular risk factors for ECAS, ICAS, SLI, and WMH.

\begin{tabular}{|c|c|c|c|c|c|c|c|c|}
\hline & \multicolumn{2}{|c|}{ ECAS } & \multicolumn{2}{|l|}{ ICAS } & \multicolumn{2}{|l|}{ SLI } & \multicolumn{2}{|l|}{ WMH } \\
\hline & OR $(95 \% \mathrm{Cl})$ & $P$ & OR (95\% Cl) & $P$ & OR (95\% Cl) & $P$ & OR (95\% Cl) & $P$ \\
\hline Gender, female & 1.09 (0.68-1.75) & 0.711 & $1.15(0.71-1.88)$ & 0.573 & $0.77(0.49-1.21)$ & 0.257 & 1.15 (0.79-1.69) & 0.465 \\
\hline Age & $1.43(0.93-2.19)$ & 0.103 & $2.12(1.34-3.36)$ & 0.001 & $2.64(1.71-4.06)$ & $<0.001$ & $4.95(3.44-7.11)$ & $<0.001$ \\
\hline Hypertension & $1.37(0.90-2.11)$ & 0.147 & $1.92(1.21-3.05)$ & 0.006 & $2.12(1.38-3.26)$ & 0.001 & $1.74(1.24-2.45)$ & 0.001 \\
\hline Diabetes mellitus & $1.80(1.17-2.77)$ & 0.007 & $1.10(0.69-1.75)$ & 0.685 & $1.15(0.75-1.76)$ & 0.521 & $1.04(0.72-1.51)$ & 0.839 \\
\hline Current smoking & $1.15(0.65-2.02)$ & 0.632 & 1.56 (0.89-2.73) & 0.123 & $1.84(1.11-3.06)$ & 0.018 & $1.33(0.83-2.12)$ & 0.232 \\
\hline CAOD & $1.21(0.61-2.40)$ & 0.584 & $1.84(0.96-3.52)$ & 0.066 & $0.69(0.33-1.43)$ & 0.317 & $0.89(0.50-1.59)$ & 0.695 \\
\hline CKD & $1.24(0.76-2.02)$ & 0.395 & $1.24(0.76-2.03)$ & 0.395 & $1.55(0.99-2.44)$ & 0.057 & $1.90(1.28-2.81)$ & 0.001 \\
\hline THR & $1.06(0.95-1.19)$ & 0.314 & $1.24(1.10-1.39)$ & 0.001 & 1.07 (0.96-1.25) & 0.140 & $1.00(0.90-1.11)$ & 0.982 \\
\hline
\end{tabular}

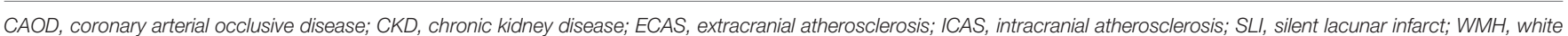
matter hyperintensity; THR, triglyceride (TG)/high-density lipoprotein cholesterol (HDL-C) ratio; OR, odds ratio; Cl, confidence interval.

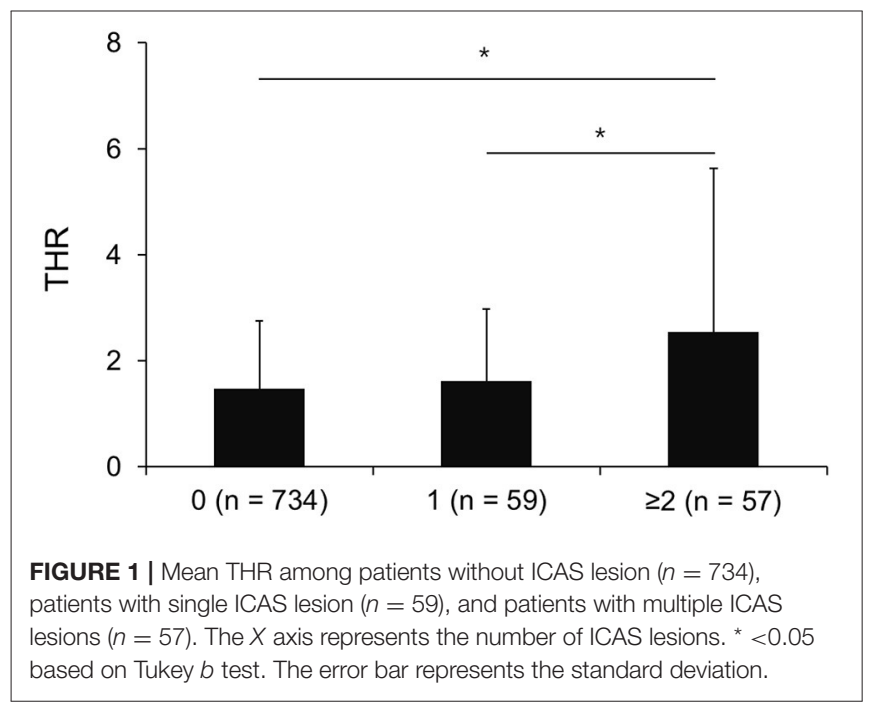

may be specifically associated with ICAS and higher risk of early recurrent stroke (24).

In addition, THR was not associated with SLI and WMHs as indices of SVD in the present study. To date, few studies have been performed within the context of observing an association between THR and SVD. In a cross-sectional study in a healthy Korean population, a positive association was observed between THR and males with silent brain infarct (25). In our population, no associations were found among THR tertiles in SLI and WMHs. Our main finding is that THR and ICAS are closely related to positive correlation. Although low HDL-C level was shown associated with WMH severity (39), the authors did not evaluate the relationship between THR and WMH. Reasons for discrepancies between previous studies and our data may be due to differences in study design and subject characteristics. In contrast to the well-known association between hyperlipidemia and LAA, the relationship between hyperlipidemia and SVD is unclear (7). SVDs were shown to have mechanistically different pathogenesis than LAA, such as lipohyalinosis, segmental demyelination, and endothelial dysfunction, indicating the nonatherogenic nature of SVDs (40). Because of the current lack of data, further studies are needed to clarify the exact association between THR and SVD.

The present study had several limitations. First, because this study was retrospective in design, a selection bias existed. Furthermore, the demographic characteristics were not the same as the general population because the study subjects visited the outpatient department for seeking underlying cardiovascular risk factors. For this reason, the possibility of selection bias was not completely excluded. Second, all study subjects were Koreans, so the results cannot be generalized to the general population. Because the prevalence of ICAS and SVD is higher in Asians than in the Western population, the results may differ across ethnicities. Third, the definition in the present study of atherosclerosis based on brain MRA did not include mild cases because of low MRA resolution. Fourth, this is a single-center study, and the sample size was too small to be generalized, requiring large-scale external validation of the current study. Fifth, statin was included in this study, but antiplatelet or anticoagulant agents were not included in the analysis. Antiplatelet or anticoagulant agents may affect atherosclerosis, so further research is needed for investigation. Finally, serum LDL-C levels and body mass index, which have a significant effect on THR and atherosclerosis, were not identified. Although serum LDL-C level is an established risk factor for coronary heart disease, the association between LDL-C and cerebral atherosclerosis is controversial (41). In a retrospective analysis of patients with ischemic stroke or transient ischemic attack, an elevated level of serum THR, but not LDL-C, was associated with large artery atherosclerotic stroke in close agreement with the results of our study (41). In addition, THR was shown a better predictor of LDL particle size than LDL-C in nondiabetic patients $(18,37,42,43)$. Therefore, LDL-C levels are less likely to affect the association between THR and ICAS. A firm conclusion could not be drawn based on results from the present study; thus, further prospective observations conducted on the general population are necessary to validate the results. 


\section{CONCLUSION}

THR was independently correlated with ICAS. Conversely, THR did not show any significant value for diagnosis of other cerebrovascular pathologies. Based on the study results, THR can be used as a feasible, efficient, routine, immediately obtainable, and inexpensive biomarker for ICAS. Because various treatment strategies necessary to reduce the risk of ischemic stroke depend on different cerebrovascular pathologies, further studies are needed, and nonstatin lipid-lowering agents for increasing HDL$\mathrm{C}$ and reducing TG could be a promising treatment strategy for prevention of ICAS-related stroke.

\section{DATA AVAILABILITY STATEMENT}

All datasets generated for this study are included in the article/supplementary material.

\section{ETHICS STATEMENT}

The studies involving human participants were reviewed and approved by The Institutional Review Board (IRB) of CHA Bundang Medical Center approved the study

\section{REFERENCES}

1. Amarenco P, Bogousslavsky J, Caplan LR, Donnan GA, Hennerici MG. Classification of stroke subtypes. Cerebrovasc Dis. (2009) 27:493501. doi: 10.1159/000210432

2. Feldmann E, Daneault N, Kwan E, Ho KJ, Pessin MS, Langenberg P, et al. Chinese-white differences in the distribution of occlusive cerebrovascular disease. Neurology. (1990) 40:1541-5. doi: 10.1212/WNL.40.10.1540

3. Gorelick PB, Caplan LR, Hier DB, Parker SL, Patel D. Racial differences in the distribution of anterior circulation occlusive disease. Neurology. (1984) 34:54-9. doi: 10.1212/WNL.34.1.54

4. Kim JS, Nah HW, Park SM, Kim SK, Cho KH, Lee J, et al. Risk factors and stroke mechanisms in atherosclerotic stroke: intracranial compared with extracranial and anterior compared with posterior circulation disease. Stroke. (2012) 43:3313-8. doi: 10.1161/STROKEAHA.112.658500

5. Vermeer SE, Hollander M, van Dijk EJ, Hofman A, Koudstaal PJ, Breteler $\mathrm{MM}$, et al. Silent brain infarcts and white matter lesions increase stroke risk in the general population: the Rotterdam Scan Study. Stroke. (2003) 34:1126-9. doi: 10.1161/01.STR.0000068408.82115.D2

6. Fisher CM. The arterial lesions underlying lacunes. Acta Neuropathol. (1968) 12:1-15. doi: 10.1007/BF00685305

7. Aboyans V, Lacroix P, Criqui MH. Large and small vessels atherosclerosis: similarities and differences. Prog Cardiovasc Dis. (2007) 50:112-25. doi: 10.1016/j.pcad.2007.04.001

8. Colhoun HM, Betteridge DJ, Durrington PN, Hitman GA, Neil HA, Livingstone SJ, et al. Primary prevention of cardiovascular disease with atorvastatin in type 2 diabetes in the collaborative atorvastatin diabetes study (CARDS): multicentre randomised placebo-controlled trial. Lancet. (2004) 364:685-96. doi: 10.1016/S0140-6736(04)16895-5

9. Amarenco P, Bogousslavsky J, Callahan A 3rd, Goldstein LB, Hennerici $\mathrm{M}$, Rudolph AE, et al. High-dose atorvastatin after stroke or transient ischemic attack. N Engl J Med. (2006) 355:549-59. doi: 10.1056/NEJMoa 061894

10. Tanne D, Koren-Morag N, Graff E, Goldbourt U. Blood lipids and firstever ischemic stroke/transient ischemic attack in the bezafibrate infarction prevention (BIP) registry: high triglycerides constitute an independent risk factor. Circulation. (2001) 104:2892-7. doi: 10.1161/hc4901.100384
(IRB no. BD-2010-083). The patients/participants provided their written informed consent to participate in this study.

\section{AUTHOR CONTRIBUTIONS}

M-HW and KOL: conception, design, drafting of manuscript, acquisition of data, final approval of manuscript. DC: design, acquisition of data, revision of manuscript, final approval of manuscript. J-WC: design, acquisition of data, revision of manuscript, final approval of manuscript. S-HK: design, acquisition of data, interpretation of data, revision of manuscript, final approval of manuscript. S-HO: conception, design, analysis and interpretation of data, drafting of the manuscript, revision of manuscript, final approval of manuscript. All authors contributed to the article and approved the submitted version.

\section{FUNDING}

This study was supported by a grant of Basic Science Research Program through National Research Foundation of Korea (NRF) funded by the Ministry of Education (NRF2018R1D1A1B07050579).
11. Bansal S, Buring JE, Rifai N, Mora S, Sacks FM, Ridker PM. Fasting compared with nonfasting triglycerides and risk of cardiovascular events in women. JAMA. (2007) 298:309-16. doi: 10.1001/jama.298.3.309

12. Kurth T, Everett BM, Buring JE, Kase CS, Ridker PM, Gaziano JM. Lipid levels and the risk of ischemic stroke in women. Neurology. (2007) 68:55662. doi: 10.1212/01.wnl.0000254472.41810.0d

13. Wannamethee SG, Shaper AG, Ebrahim S. HDL-Cholesterol, total cholesterol, and the risk of stroke in middle-aged British men. Stroke. (2000) 31:18828. doi: 10.1161/01.STR.31.8.1882

14. Lindenstrom E, Boysen G, Nyboe J. Influence of total cholesterol, high density lipoprotein cholesterol, and triglycerides on risk of cerebrovascular disease: the copenhagen city heart study. BMJ. (1994) 309:11-5. doi: 10.1136/bmj.309.6946.11

15. Fruchart JC, Sacks F, Hermans MP, Assmann G, Brown WV, Ceska R, et al. The residual risk reduction initiative: a call to action to reduce residual vascular risk in patients with dyslipidemia. Am J Cardiol. (2008) 102 (Suppl. 10):1K-34K. doi: 10.1016/j.amjcard.2008.10.002

16. Millan J, Pinto X, Munoz A, Zuniga M, Rubies-Prat J, Pallardo LF, et al. Lipoprotein ratios: physiological significance and clinical usefulness in cardiovascular prevention. Vasc Health Risk Manag. (2009) 5:75765. doi: 10.2147/VHRM.S6269

17. Boizel R, Benhamou PY, Lardy B, Laporte F, Foulon T, Halimi S. Ratio of triglycerides to HDL cholesterol is an indicator of LDL particle size in patients with type 2 diabetes and normal HDL cholesterol levels. Diabetes Care. (2000) 23:1679-85. doi: 10.2337/diacare.23.11.1679

18. Bhalodkar NC, Blum S, Enas EA. Accuracy of the ratio of triglycerides to high-density lipoprotein cholesterol for predicting low-density lipoprotein cholesterol particle sizes, phenotype B, and particle concentrations among Asian Indians. Am J Cardiol. (2006) 97:1007-9. doi: 10.1016/j.amjcard.2005.10.036

19. Dobiasova M, Frohlich J. The plasma parameter log (TG/HDL-C) as an atherogenic index: correlation with lipoprotein particle size and esterification rate in apoB-lipoprotein-depleted plasma (FER(HDL)). Clin Biochem. (2001) 34:583-8. doi: 10.1016/S0009-9120(01)00263-6

20. Pacifico L, Bonci E, Andreoli G, Romaggioli S, Di Miscio R, Lombardo $\mathrm{CV}$, et al. Association of serum triglyceride-to-HDL cholesterol ratio with carotid artery intima-media thickness, insulin resistance and nonalcoholic 
fatty liver disease in children and adolescents. Nutr Metab Cardiovasc Dis. (2014) 24:737-43. doi: 10.1016/j.numecd.2014.01.010

21. Salazar MR, Carbajal HA, Espeche WG, Aizpurua M, Maciel PM, Reaven GM. Identification of cardiometabolic risk: visceral adiposity index versus triglyceride/HDL cholesterol ratio. Am J Med. (2014) 127:1527. doi: 10.1016/j.amjmed.2013.10.012

22. Deng QW, Wang H, Sun CZ, Xing FL, Zhang HQ, Zuo L, et al. Triglyceride to high-density lipoprotein cholesterol ratio predicts worse outcomes after acute ischaemic stroke. Eur J Neurol. (2017) 24:283-91. doi: 10.1111/ene.13198

23. Kang K, Lee K, Chung SH. The triglyceride:high-density lipoproteincholesterol ratio and steno-occlusive disease in the intracranial arteries. $J$ Thromb Thrombolysis. (2011) 32:103-9. doi: 10.1007/s11239-011-0553-1

24. Sirimarco G, Deplanque D, Lavallee PC, Labreuche J, Meseguer E, Cabrejo L, et al. Atherogenic dyslipidemia in patients with transient ischemic attack. Stroke. (2011) 42:2131-7. doi: 10.1161/STROKEAHA. 110.609727

25. Nam KW, Kwon HM, Jeong HY, Park JH, Kwon H, Jeong SM. High triglyceride/HDL cholesterol ratio is associated with silent brain infarcts in a healthy population. BMC Neurol. (2019) 19:147. doi: 10.1186/s12883-019-1373-8

26. North American Symptomatic Carotid Endarterectomy Trial Collaborators, Barnett HJM, Taylor DW, Haynes RB, Sackett DL, Peerless SJ, et al. Beneficial effect of carotid endarterectomy in symptomatic patients with high-grade carotid stenosis. N Engl J Med. (1991) 325:445-53. doi: 10.1056/NEJM199108153250701

27. Chimowitz MI, Lynn MJ, Howlett-Smith H, Stern BJ, Hertzberg VS, Frankel MR, et al. Comparison of warfarin and aspirin for symptomatic intracranial arterial stenosis. $N$ Engl J Med. (2005) 352:1305-16. doi: 10.1056/NEJMoa043033

28. Fazekas F, Chawluk JB, Alavi A, Hurtig HI, Zimmerman RA. MR signal abnormalities at $1.5 \mathrm{~T}$ in Alzheimer's dementia and normal aging. AJR Am J Roentgenol. (1987) 149:351-6. doi: 10.2214/ajr.149.2.351

29. D’Armiento FP, Bianchi A, de Nigris F, Capuzzi DM, D’Armiento MR, Crimi G, et al. Age-related effects on atherogenesis and scavenger enzymes of intracranial and extracranial arteries in men without classic risk factors for atherosclerosis. Stroke. (2001) 32:2472-9. doi: 10.1161/hs1101. 098520

30. Barter PJ, Nicholls S, Rye KA, Anantharamaiah GM, Navab M, Fogelman AM. Antiinflammatory properties of HDL. Circ Res. (2004) 95:76472. doi: 10.1161/01.RES.0000146094.59640.13

31. Lopez-Cancio E, Galan A, Dorado L, Jimenez M, Hernandez $\mathrm{M}$, Millan $\mathrm{M}$, et al. Biological signatures of asymptomatic extra- and intracranial atherosclerosis: the Barcelona-AsIA (asymptomatic intracranial atherosclerosis) study. Stroke. (2012) 43:2712-9. doi: 10.1161/STROKEAHA.112.661702

32. Park JH, Kwon HM, Roh JK. Metabolic syndrome is more associated with intracranial atherosclerosis than extracranial atherosclerosis. Eur J Neurol. (2007) 14:379-86. doi: 10.1111/j.1468-1331.2007.01682.x

33. Bang OY, Kim JW, Lee JH, Lee MA, Lee PH, Joo IS, et al. Association of the metabolic syndrome with intracranial atherosclerotic stroke. Neurology. (2005) 65:296-8. doi: 10.1212/01.wnl.0000168862.09764.9f
34. Palmieri VO, Grattagliano I, Portincasa P, Palasciano G. Systemic oxidative alterations are associated with visceral adiposity and liver steatosis in patients with metabolic syndrome. J Nutr. (2006) 136:3022-6. doi: 10.1093/jn/136.12.3022

35. Park HY, Kyeong H, Park DS, Lee HS, Chang H, Kim YS, et al. Correlation between insulin resistance and intracranial atherosclerosis in patients with ischemic stroke without diabetes. J Stroke Cerebrovasc Dis. (2008) 17:4015. doi: 10.1016/j.jstrokecerebrovasdis.2008.06.004

36. Laws A, Reaven GM. Evidence for an independent relationship between insulin resistance and fasting plasma HDL-cholesterol, triglyceride and insulin concentrations. J Intern Med. (1992) 231:25-30. doi: 10.1111/j.1365-2796.1992.tb00494.x

37. McLaughlin T, Reaven G, Abbasi F, Lamendola C, Saad M, Waters D, et al. Is there a simple way to identify insulin-resistant individuals at increased risk of cardiovascular disease? Am J Cardiol. (2005) 96:399404. doi: 10.1016/j.amjcard.2005.03.085

38. Carr MC, Brunzell JD. Abdominal obesity and dyslipidemia in the metabolic syndrome: importance of type 2 diabetes and familial combined hyperlipidemia in coronary artery disease risk. J Clin Endocrinol Metab. (2004) 89:2601-7. doi: 10.1210/jc.2004-0432

39. Crisby M, Bronge L, Wahlund LO. Low levels of high density lipoprotein increase the severity of cerebral white matter changes: implications for prevention and treatment of cerebrovascular diseases. Curr Alzheimer Res. (2010) 7:534-9. doi: 10.2174/156720510792231694

40. Wardlaw JM, Smith C, Dichgans M. Small vessel disease: mechanisms and clinical implications. Lancet Neurol. (2019) 18:684-96. doi: 10.1016/S1474-4422(19)30079-1

41. Bang OY, Saver JL, Liebeskind DS, Pineda S, Ovbiagele B. Association of serum lipid indices with large artery atherosclerotic stroke. Neurology. (2008) 70:841-7. doi: 10.1212/01.wnl.0000294323.48661.a9

42. Fan X, Liu EY, Hoffman VP, Potts AJ, Sharma B, Henderson DC. Triglyceride/high-density lipoprotein cholesterol ratio: a surrogate to predict insulin resistance and low-density lipoprotein cholesterol particle size in nondiabetic patients with schizophrenia. J Clin Psychiatry. (2011) 72:80612. doi: $10.4088 / \mathrm{JCP} .09 \mathrm{~m} 05107 \mathrm{yel}$

43. Hanak V, Munoz J, Teague J, Stanley A, Jr., Bittner V. Accuracy of the triglyceride to high-density lipoprotein cholesterol ratio for prediction of the low-density lipoprotein phenotype B. Am J Cardiol. (2004) 94:21922. doi: $10.1016 /$ j.amjcard.2004.03.069

Conflict of Interest: The authors declare that the research was conducted in the absence of any commercial or financial relationships that could be construed as a potential conflict of interest.

Copyright (๑) 2021 Woo, Lee, Chung, Choi, Kim and Oh. This is an open-access article distributed under the terms of the Creative Commons Attribution License (CC $B Y)$. The use, distribution or reproduction in other forums is permitted, provided the original author(s) and the copyright owner(s) are credited and that the original publication in this journal is cited, in accordance with accepted academic practice. No use, distribution or reproduction is permitted which does not comply with these terms. 